\title{
Manuel REBUSCHI, Qu'est-ce que la signification?
}

Paris, J. Vrin, coll. Chemins philosophiques, 2008, 125 p.

\section{Marion Duvauchel}

\section{OpenEdition}

\section{Journals}

\section{Édition électronique}

URL : http://journals.openedition.org/questionsdecommunication/242

DOI : 10.4000/questionsdecommunication.242

ISSN : 2259-8901

\section{Éditeur}

Presses universitaires de Lorraine

\section{Édition imprimée}

Date de publication : 30 juin 2010

ISBN : 978-2-8143-0024-8

ISSN : 1633-5961

\section{Référence électronique}

Marion Duvauchel, «Manuel ReBuschl, Qu'est-ce que la signification? », Questions de communication [En ligne], 17 | 2010, mis en ligne le 23 janvier 2012, consulté le 22 septembre 2020. URL : http:// journals.openedition.org/questionsdecommunication/242 ; DOI : https://doi.org/10.4000/ questionsdecommunication.242

Ce document a été généré automatiquement le 22 septembre 2020

Tous droits réservés 


\section{Manuel REBUSCHI, Qu'est-ce que la signification?}

Paris, J. Vrin, coll. Chemins philosophiques, 2008, 125 p.

\section{Marion Duvauchel}

\section{RÉFÉRENCE}

Manuel REBUSCHI, Qu'est-ce que la signification? Paris, J. Vrin, coll. Chemins

philosophiques, 2008, $125 \mathrm{p}$.

1 La librairie philosophique Jacques Vrin a un grand souci pédagogique. Qu'est-ce que la signification? en témoigne. Destiné aux étudiants des universités et des classes préparatoires, le livre n'oublie pas non plus un public dit cultivé et qui l'est sûrement puisqu'il attend, si l'on en croit la quatrième de couverture, " un traitement direct et clair de questions de philosophie générale ». Quoi de plus général en effet que la notion de signification qui, si elle ne fait pas partie des quelques quinze grandes notions du programme de philosophie de terminale, est tout de même au cœur de ce qu'on appelle - en philosophie - l'herméneutique, c'est-à-dire l'interprétation, et participe des interrogations sur le langage. Sur cette même quatrième de couverture où les intentions de l'ouvrage et sans doute de l'auteur sont affichées, on peut lire les quatre questions que l'on peut considérer comme centrales et que l'on va s'attaquer à résoudre: y a-t-il un intermédiaire entre le langage et le monde? La signification se réduit-elle à l'information? Les significations sont-elles dans la tête? Les formalismes peuvent-ils expliquer la signification? Hélas, aucune de ces questions n'est véritablement traitée. C'est bien dommage car chacune est des plus pertinentes. Avec la première, c'est la question des rapports entre la pensée et le langage qui est posée. En effet, voit-on un autre intermédiaire entre le monde et la langue que la pensée? Hegel a affronté la question dans la langue un peu surnaturelle qui est la sienne. On s'accorde aujourd'hui assez simplement et avec lui à admettre la réciproque rétroaction de l'une sur l'autre, et si c'est à peu près tout, encore convenait-il de le signaler puisque ce livre 
s'adresse à des étudiants. La deuxième question est dans l'air du temps. Communiquer, est-ce informer? Si ce n'était que cela, la vie serait sinistre et le langage réduit à sa seule fonction instrumentale. L'usage et l'élégance intellectuelle veulent que l'on procède dans ce cas à une petite étude de l'aire sémantique de chacun des termes que l'on emploie. C'est une manière de reconnaître l'épaisseur et la chair de la langue, une manière de ne pas oublier que les mots ont une histoire, qu'ils se chargent de significations à travers les siècles. Tout le contraire de la formalisation mathématique qui consiste à changer un mot en un " $\mathrm{X}$ ", et d'entrer ainsi dans la formidable modélisation logique, efficace, puissante, redoutable et décharnalisante. Quant à la troisième question de savoir où se logent les significations, elle n'est pas seulement un mythe décoratif ou une question dépassée. Les scolastiques se la posaient déjà, et c'est au cœur de toute épistémologie. La connaissance est-elle dans l'objet connu, dans le sujet connaissant ou dans l'interaction entre les deux? Jean Piaget a donné quelques éléments de réponse que les formalistes feraient bien de ne pas ignorer ou de feindre d'ignorer. Il n'aimait guère la philosophie, et sans doute les philosophes, il est vrai. On peut considérer que cet opuscule tente de répondre à la quatrième question, celle de savoir si les formalismes peuvent expliquer la signification. Or, sans vraiment en avoir le projet, il montre que, pour répondre à la question de la signification, les formalistes ont modélisé de telle sorte qu'elle soit occultée, et c'est l'histoire de ce refoulement comme diraient les psychanalystes - que, sans le savoir, il raconte. À quoi s'emploie alors l'auteur? À montrer sa culture et à exposer les modèles qui ont dominé la question tout en se gardant bien d'y répondre et en mettant hors-jeu un certain nombre de faits décisifs, à commencer par la dimension cognitive du sens qui est abandonnée pour des modèles purement formels. L'auteur va même jusqu'à formuler l'idée, parfaitement vraie, selon laquelle « la philosophie du langage s'est fourvoyée dès le départ en glissant sur la pente savonneuse des méthodes logico-formelles, alors que les approches structuralistes ou mentalistes pratiquées par les linguistes offraient un cadre alternatif cohérent pour penser la signification» (p. 80). Mais c'est pour y répondre aussitôt : "Une réponse plus subtile consistera à affiner le formalisme plutôt qu'à jeter le bébé formel avec l'eau du bain » (ibid.). Là, il faut admirer la tournure impersonnelle du style. On frissonne devant tant de courage intellectuel. L'autre problème que pose l'analyse logique du langage est tout aussi clairement formulé : « Ce qui est mis à l'épreuve au travers le l'analyse logique du langage, ce [...] sont des présuppositions ontologiques ou métaphysiques générales, en particulier nos conceptions du possible et du nécessaire»( (p. 8). Absolument! Cela méritait d'être traité, avec la question du probable, qui est au cœur des relations de significations. Le premier paragraphe du livre commençait pourtant plutôt bien: de quoi s'agit-il lorsqu'on parle de signification? On nous dit avec justesse que «nous parlons du monde» (p. 7), ce qui est banal. Mais par là, il faut entendre que lorsque nous parlons nous parlons de quelque chose - du moins si nous souhaitons être écouté. On peut aussi, il est vrai, ne parler de rien, ou parler du rien de la langue, à la Maurice Blanchot. On s'en lasse vite, comme de la nouvelle cuisine. Nous parlons donc, pour Manuel Rebuschi, «mais nous ne disons pas que le vrai. Lorsque nous énonçons le faux par erreur nous échouons à parler du monde » (ibid.). Curieuse idée. Énoncer le faux par erreur, ce n'est pas nécessairement échouer à parler du monde mais échouer à en donner une représentation juste, ce qui n'est pas la même chose. De là, l'auteur en déduit que la signification déborde le seul rapport langage-monde. Ce qui est exact, mais pas du tout pour des raisons de vrai ou de faux. La signification déborde le rapport 
de vérité conçu en termes hérités de Descartes, c'est-à-dire en termes binaires de vrai ou de faux, ce que l'auteur semble ignorer. Avec Descartes, c'est un changement de paradigme qui se met en place et la recherche de la vérité ne s'entend plus comme quête de la sagesse, ou dans l'horizon de la sagesse et de la justice, mais comme distinction du vrai et du faux. N'importe quel professeur de philosophie de l'enseignement secondaire est capable de l'expliquer. La philosophie analytique et tous les formalismes se sont tout simplement installés dans un paradigme, et avec d'autant plus de puissance que toute une société technicienne ne jurait que par les sciences dites dures et leur attribuait un statut qu'elle revendiquait pour elle seule. On a ici une petite histoire des modèles philosophiques successifs de 1969 à 1980 dans lesquels la signification a été abordée, en bref, les doctrines de la signification qui se sont imposées jusqu'à aujourd'hui. Ce n'est pas sans intérêt, encore fallait-il l'annoncer comme tel. À partir de là, quelques questions se posent encore. L'une des interrogations que soulèvent les modèles analytiques est celle de la différence entre la langue naturelle et le langage formel. Au fur et à mesure que la philosophie du langage s'est préoccupée de modéliser les questions liées à la langue, elle a construit un système de logique sémantique. Non pour comprendre la signification mais, au contraire, pour fournir un modèle logique susceptible d'en rendre compte, au prix d'une terrible restriction et surtout au prix d'un refus de reconnaitre une différence entre la langue naturelle et un langage formel. Le bon sens le plus élémentaire, que nous ne sommes pas tenus d'abandonner au motif que nous philosophons, impose pourtant cette idée que l'interprétation d'un texte n'implique pas seulement des catégories logiques mais des catégories linguistiques, qui renvoient elles-mêmes à des catégories ontologiques, des catégories de l'être. Tout ce qui déborde du texte et qui renvoie au monde renvoie aussi à cette catégorie particulière du réel que la logique ne peut prendre en compte et qui s'appelle le temps. Le monde référentiel n'est pas seulement le monde des choses, mais celui de la durée, de la temporalité. Pour modéliser, il faut exclure deux choses, la dimension cognitive et la dimension temporelle. Or, si pour Gottlob Frege, le premier de la liste, la signification relève de la référence (on parle de quelque chose), les penseurs qui vont suivre ne se soucient plus de référence. Le postulat de Gottlob Frege est celui d'un sens objectif. La faiblesse de celui-ci est certes bien soulignée : l'univers de la fiction est déclaré hors-jeu (p. 19). Or, tout le champ de la littérature est de la fiction. La théorie de Gottlob Frege ne peut gérer des énoncés comme «Shrek est parti en vacances » (p. 20), ou plus simplement « Le père Goriot aime ses deux filles ». Avec Bertrand Russell, on sait d'emblée à quoi on s'expose : son approche vise à résoudre des problèmes laissés en suspens par Gottlob Frege, "la possibilité de la vérité des assertions existentielles négatives, et l'universalité du tiers-exclu» (p. 21). On voit toute l'extension philosophique des approches sémantiques. Elles ne sont que des expansions en philosophie du formalisme mathématique. Le plus navrant, c'est que ces excroissances se sont imposées aujourd'hui comme l'horizon de la philosophie. Nous n'en sommes qu'à la page 23 et tout cela donne l'impression d'un discours pour initiés, de préférence ayant fait une terminale $\mathrm{S}$. Ici et là, on a quelques phrases éclairantes, un peu de lumière dans cet entrelacs de formules, de logarithmes, etc. Gottlob Frege, Bertrand Russell et Willard van Orman Quine s'inscrivent dans un modèle universaliste où le langage est conçu comme unique, inamovible tout ce qui est exprimable s'y exprime et rien de ce qui lui échappe n'est exprimable (p.37). Le postulat des deux hommes est difficilement recevable et cependant aucune critique n'en est formulée. Le langage n'est pas la seule source d'expression. Dès les premiers modèles, les 
philosophes analytiques excluent ainsi de l'univers de l'expression - et donc de l'univers de la signification - le mime Marceau, la Moldau, et la fonction alpha de Bion. Cela fait beaucoup. Ce seul présupposé aurait dû être dénoncé avec l'enseignement des théories de ces « matheux » qui ont prétendu imposer les normes des mathématiques à l'univers du sens, en le réduisant d'autant mieux. Sans doute que cela les a rassurés. Le modèle analytique suivant est celui où le langage est « conçu comme calcul». En soi, cela devrait nous faire blêmir. Quand on compte, on ne peut guère interpréter. Cet opuscule réussit à nous livrer l'histoire des avatars d'une pensée fondée sur des présupposés faux sans en faire jamais la moindre critique. Pourtant, le problème est bien soulevé avec Alfred Tarski, « qui ne donne pas une théorie de la signification mais de la vérité » (p. 37). Une fois qu'on a montré qu'un présupposé est faux à quoi bon continuer à raisonner? À la question «qu'est-ce que la signification? », les modèles analytiques ont répondu en la soumettant à un ordre purement logique qui ne peut en rendre compte. Et à aucun moment, cela n'est analysé. En effet, les approches structuralistes fournissaient un cadre cohérent permettant de penser la signification, ce que les approches analytiques ne permettent pas. Le dire, c'est l'honnêteté intellectuelle et même ce qu'exige la vérité. Un livre demande un minimum d'honnêteté : à commencer par le respect du rapport annoncé entre un titre et un contenu. Depuis les années 80, "les travaux ont conduit au développement de la sémantique des situations, de la théorie des représentations de discours et de la logique dynamique » (p. 81).

\section{AUTEURS}

\section{MARION DUVAUCHEL}

Lycée français d'Agadir, Maroc

Marie-France.Duvauchel@yahoo.fr 